\title{
Broadband Characterisation of Interior Materials and Surface Scattering using Terahertz Time-Domain Spectroscopy
}

\author{
Emily Adams, Toby Attwood, Suzanna Freer \\ School of Physics and Astronomy \\ University of Birmingham \\ Birmingham, United Kingdom \\ \{ECA725, TXA740,SXF845\}@ student.bham.ac.uk
}

\author{
Fangjing $\mathrm{Hu}$ \\ School of Physics \\ Huazhong University of Science and Technology \\ Wuhan, China \\ fangjing_hu@hust.edu.cn
}

\author{
Stephen M. Hanham, Costas Constantinou \\ Department of Electronic, Electrical and Systems Engineering \\ University of Birmingham \\ Birmingham, United Kingdom \\ \{s.hanham, c.constantinou\}@bham.ac.uk
}

Leyre Azpilicueta

School of Engineering and Sciences

Tecnologico de Monterrey

Monterrey, Mexico

leyre.azpilicueta@tec.mx

\author{
Miguel Navarro-Cía \\ School of Physics and Astronomy \& School of Engineering \\ University of Birmingham \\ Birmingham, United Kingdom \\ m.navarro-cia@bham.ac.uk
}

\begin{abstract}
Indoor wireless communications need to move towards Terahertz (THz) frequencies in order to keep up with society's demand for data transmission, but this change is currently hindered by limited knowledge of material properties and propagation and scattering models at these frequencies. The dielectric properties of common household materials are investigated here with a twofold objective: (1) to extend the library of material properties at THz, and (2) to estimate and disentangle losses in scattering measurements in order to facilitate propagation, scattering and, ultimately, channel models.
\end{abstract}

Index Terms-terahertz time-domain spectroscopy, retrieval method, scattering.

\section{INTRODUCTION}

Terahertz (THz) technology [1] has applications in multiple instances of state-of-the-art research, including radar for driverless vehicles [2], and security screening [3]. Additionally, THz waves are being considered as future communication channels in indoor environments to supplement the $\mathrm{GHz}$ frequencies currently used; however, this presents a complex set of challenges.

Firstly, the properties of materials are not well documented at $\mathrm{THz}$ frequencies. This currently makes it difficult to predict dielectric losses upon reflection from an interface, or how a wave would propagate through a given dielectric. Secondly, scattering from a rough interface causes problems for the propagation of communication channels due to the potential for diffuse scattering components to interfere with the desired wave packets.
In the context of indoor wireless communications, it is challenging to have propagation, scattering, and channel models that overcome the problems stated above without a thorough investigation of material properties at $\mathrm{THz}$ frequencies.

\section{EXPERIMENTAL SETUP}

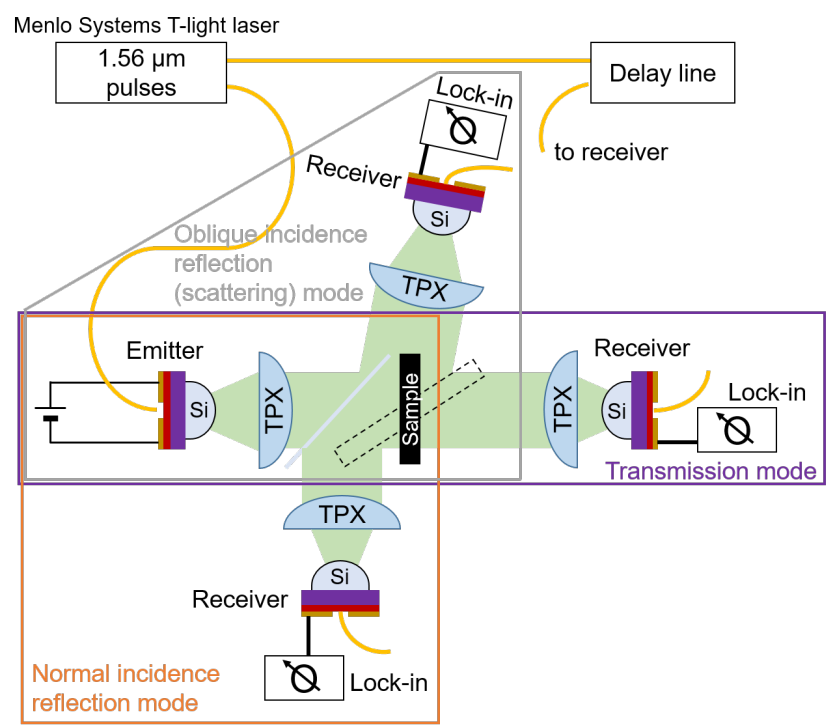

Fig. 1. Schematic diagrams of the THz-TDS system used for the material property retrieval (purple and orange boxes), and scattering measurements (grey box). 

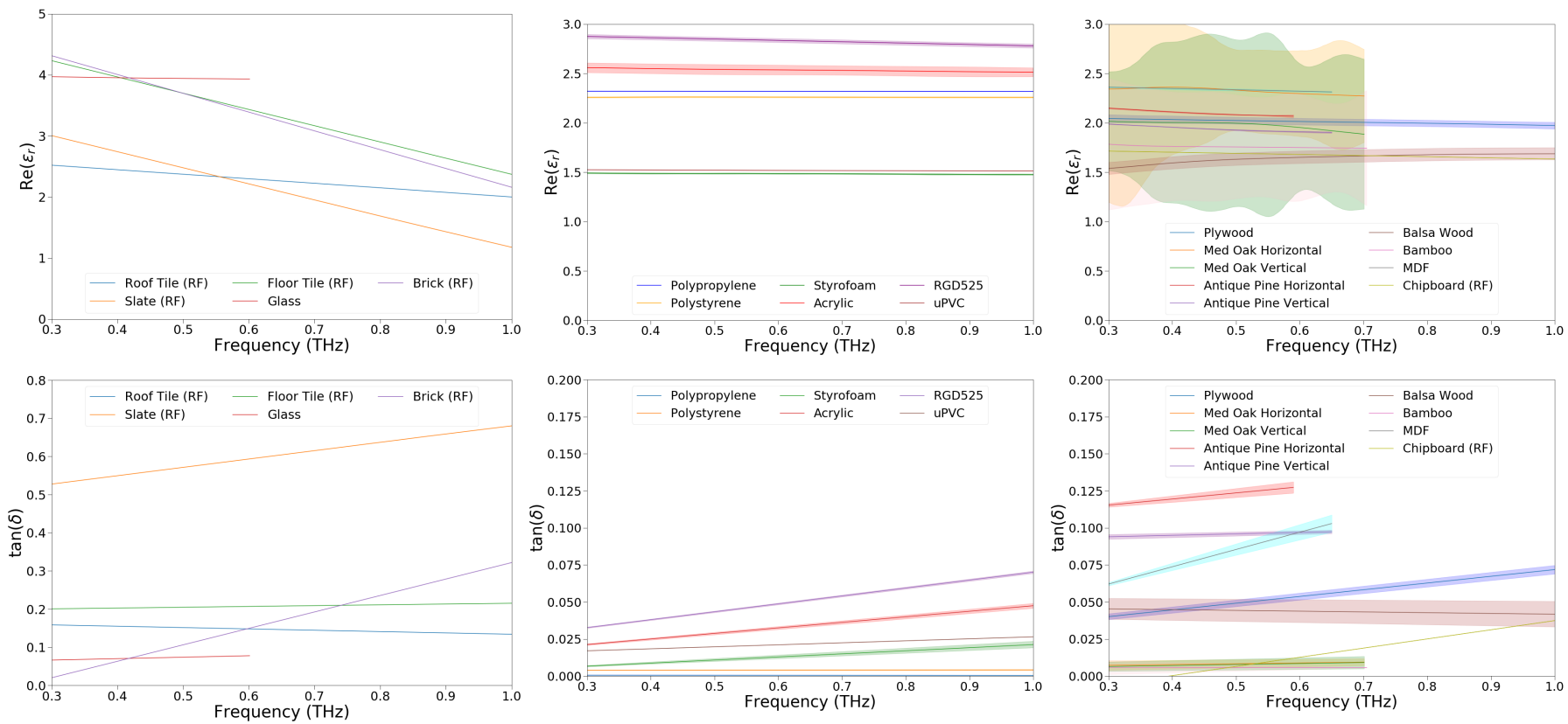

Fig. 2. Relative real part of the electric permittivity and loss tangent as a function of frequency for building materials (left), plastics (centre), and wood (right). The shaded regions indicate the error for each material. Samples whose material properties were retrieved in reflection shows (RF) in the legends.

A Menlo Systems TERA K15 THz time-domain spectrometer (THz-TDS), was used for the retrieval of material properties and for the scattering measurements. Figure 1 shows a schematic of the experimental setup with the three different configurations used in this work: transmission [4] and normal incidence reflection mode [5] for material properties extraction and oblique incidence reflection mode for scattering measurements [6].

\section{RESULTS AND DISCUSSION}

\section{A. Dielectric Properties}

For those samples with good transmission, the software Teramat or TeraLyzer was used for material properties extraction. The former was used for etalon (i.e. Fabry-Pérot) free samples, whereas the latter was used for samples displaying etalon effects. Further details about the methodology can be found in [7]. Meanwhile, for thick lossy samples unsuitable for transmission measurements, an in-house Kramers-Kronig retrieval reflection algorithm was used. Details of the extraction algorithm can be found in [5]. In addition, the averaged results (to decrease systematic errors, each sample was measured 4 times) were smoothed using the signal.savgol_filter function from the Python SciPy package.

The building materials group in Fig. 2 had the largest values of $\operatorname{Re}(\varepsilon)$ and $\tan (\delta)$, suggesting that transmission of signals through different rooms and floors would be the most weakened in the context of transmission through dielectrics. Useful data for glass could only be extracted between 0.3 $0.6 \mathrm{THz}$, as beyond this the data was too noisy.

For plastics, $\operatorname{Re}(\varepsilon)$ ranges from 1.5 to 2.85 with very low dispersion, and $\tan (\delta)$ is $<0.075$ for all cases. The acrylic and RGD525 polymers, which are common materials used for
3D printing, have the highest values of $\operatorname{Re}(\varepsilon)$ of the plastics investigated. This is good for device miniaturisation, but they also show the highest $\tan (\delta)$.

In the case of wood, the values of $\operatorname{Re}(\varepsilon)$ are similar to those of the plastics, but $\tan (\delta)$ generally tends to be larger. Some wood samples were visually anisotropic (i.e grains visible in the wood), but this does not result in a significant anisotropy for $\mathrm{THz}$ frequencies.

Similar trends for all type of materials (e.g. building materials, plastics and wood) have been reported in the literature [8].

\section{B. Scattering}

The dielectric properties found in Section III-A were then used to inform analytical scattering simulations based on the Beckmann-Spizzichino model, which was implemented in Python. The reader is referred to [9] for a general outline of this model, and to [10] for the correction which allows this model to account for dielectric properties.

An example of these results is shown in Fig. 3. These results can be used to separate dielectric and scattering losses as follows.

The infinite conductor peak represents the loss due to roughness only, as a perfect electric conductor has neither ohmic nor dielectric losses. The difference between the peaks of the materials and the infinite conductor gives the dielectric losses of each material. The different materials all have very similar peak values, and this was true for all material groups investigated. Since the materials form "bands", this is evidence that only a material type needs to be known to make a well informed estimate of total losses.

When waves scatter from a rough surface, only a fraction is reflected back in the specular direction. This fraction, $\Gamma_{\text {rough }}$, 


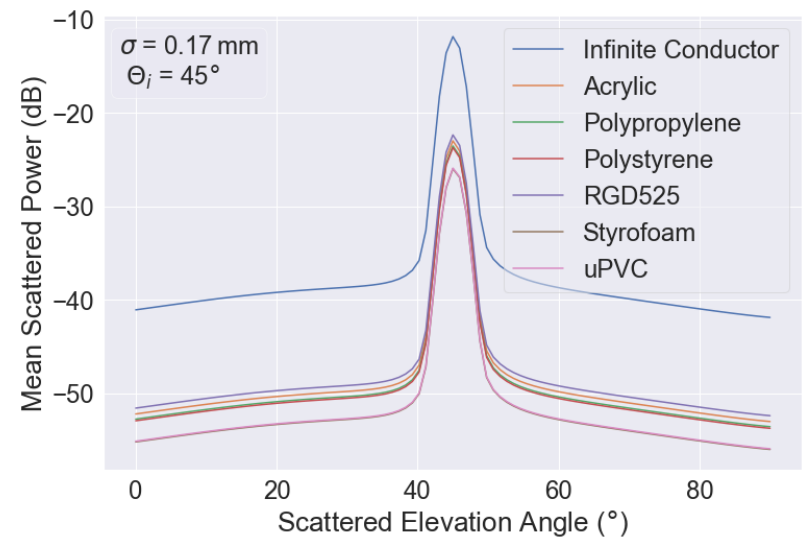

Fig. 3. Mean scattered power from each plastic surface at $300 \mathrm{GHz}$ as calculated using the Beckmann-Spizzichino model. The root mean square height is denoted by $\sigma$, and the angle of incidence, $\theta_{\mathrm{i}}=45^{\circ}$.

can be estimated by multiplying the Fresnel coefficient of a smooth surface, $\Gamma_{\text {smooth }}$, with a roughness factor, $\rho_{\mathrm{s}}$.

In the classical literature such $\rho_{\mathrm{s}}$ is the Rayleigh roughness factor [11] and is defined as

$$
\begin{aligned}
& \rho_{\mathrm{s}}=e^{\frac{-f}{2}}, \\
& \frac{f}{2}=\frac{8 \pi^{2} \sigma^{2} \cos ^{2}\left(\theta_{\mathrm{i}}\right)}{\lambda^{2}},
\end{aligned}
$$

where $\sigma$ is the root mean square height of the surface. This correction shows good fitting when the roughness parameter $g$ $<4$, as demonstrated by our experiments $\left(\theta_{\mathrm{i}}=\theta_{\mathrm{r}}=45^{\circ}\right)$ with a rough $80 \mathrm{~mm} \times 80 \mathrm{~mm}$ RGD525 sample for frequencies up to $0.4 \mathrm{THz}$ (see red dots in Fig. 4). However, for larger $g$ (i.e. above $0.4 \mathrm{THz}$ for our choice of surface roughness $\sigma=0.17$ $\mathrm{mm}$ ) the model breaks down. Motivated by this limitation, we empirically found a new roughness factor

$$
\rho_{\mathrm{s}}=2.42 \cdot \frac{f}{2} e^{\frac{-f}{2}},
$$

that fits better up to $g=9$ (i.e. up to $0.6 \mathrm{THz}$ ) as shown by the dashed red line in Fig. 4.

\section{CONCLUSION}

The dielectric properties of different indoor materials were found to inform the Python simulations of the BeckmannSpizzichino model. It was found that the power loss after scattering is very similar between materials in the same group, so informed estimates of scattering loss from a dielectric can be made using this approach. A new correction to the Rayleigh scattering factor was found empirically from experimental scattering data from a 3D printed sample of RGD525 with $\sigma=0.17 \mathrm{~mm}$. This work represents a stepping stone to pave the way for future $\mathrm{THz}$ communication and sensing services.

\section{ACKNOWLEDGMENT}

This work was supported by the Royal Society [Grant No. IES $\backslash$ R3 $\backslash 183131$ and IEC/NSFC/191104], the EPSRC [Grant Nos. EP/S018395/1 and EP/V001655/1], and the University

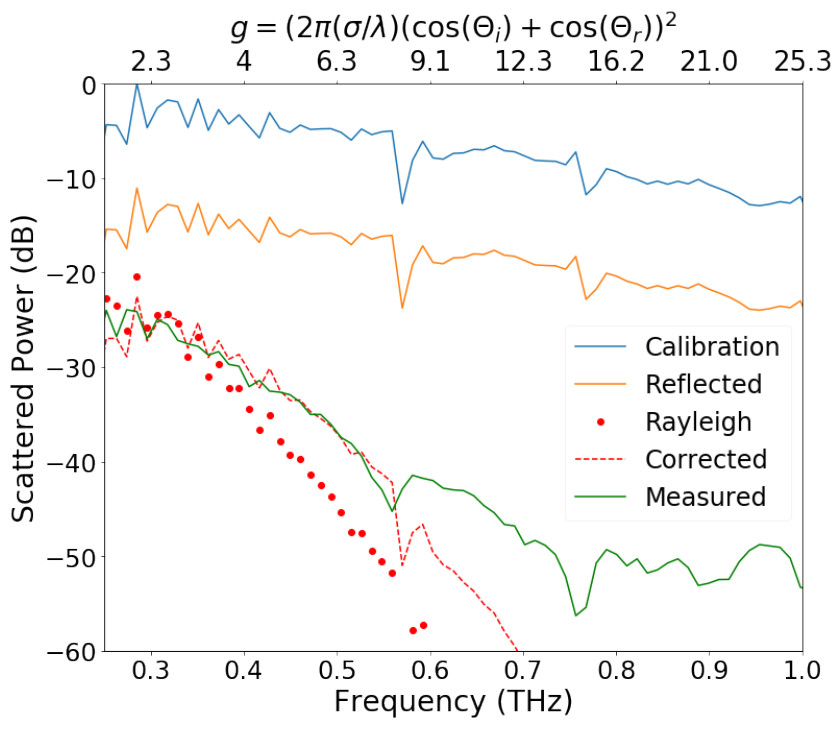

Fig. 4. Disentangled losses for a slab made of RGD525 with $\sigma=0.17 \mathrm{~mm}$ at $\theta_{\mathrm{i}}=45^{\circ}$. The corrected loss scattering factor matches up better to the measured scattering data than using the typical Rayleigh factor.

of Birmingham [PhD studentship UKRI Project Ref. 2137478, Birmingham Fellowship].

\section{REFERENCES}

[1] G. Carpintero, E. Garcia-Munoz, H. Hartnagel, S. Preu, and A. Raisanen, Semiconductor Terahertz technology: devices and systems at room temperature operation. John Wiley \& Sons, 2015.

[2] S. Minooee Sabery, A. Bystrov, M. Navarro-Cía, P. Gardner, and M. Gashinova, "Study of low terahertz radar signal backscattering for surface identification," Sensors, vol. 21, p. 2954, 2021.

[3] M. C. Kemp et al., "Security Applications of Terahertz Technology," in Terhertz for Military and Security Applications, pp. 44-52, 2003.

[4] S. Freer, A. Gorodetsky, and M. Navarro-Cía, "Beam profiling of a commercial lens-assisted terahertz time domain spectrometer," IEEE Trans. Terahertz Sci. Technol., vol. 11, no. 1, pp. 90-100, 2020.

[5] S. Freer, C. Sui, S. M. Hanham, L. Grover, and M. NavarroCía, "A Hybrid Reflection Retrieval Method for Terahertz Dielectric Imaging of Human Bone," Biomedical Optics Express, (accepted), https://doi.org/10.1364/BOE.427648.

[6] M. Camacho, R. R. Boix, S. A. Kuznetsov, M. Beruete, and M. NavarroCía, "Far-field and near-field physics of extraordinary THz transmitting hole-array antennas," IEEE Trans. Antennas Propag., vol. 67, no. 9, pp. 6029-6038, 2019.

[7] M. Ma et al., "The dielectric properties of some ceramic substrate materials at terahertz frequencies," J. Eu. Ceram. Soc., vol. 39, no. 14, pp. 4424-4428, 2019.

[8] R. Piesiewicz et al., "Properties of Building and Plastic Materials in the THz Range," Int. J. Infrared Milli. Waves, vol. 28, pp. 363-371, 2007.

[9] P. Beckmann and A. Spizzichino, The Scattering of Electromagnetic Waves from Rough Surfaces, Artech House, 1963, pp. 78-86.

[10] S. K. Nayar, K. Ikeuchi, and T. Kanade, "Surface reflection: physical and geometrical perspectives," IEEE Trans. Pattern Anal. Mach. Intell., vol. 13, no. 7, pp. 611-634, 1991.

[11] S. Ju et al. "Scattering mechanisms and modeling for terahertz wireless communications," in ICC 2019-2019 IEEE International Conference on Communications, 2019, pp. 1-7. 